\title{
A NOTE ON THE THETA CHARACTERISTICS OF A COMPACT RIEMANN SURFACE
}

\author{
INDRANIL BISWAS
}

(Received 13 January 2002; revised 5 May 2003)

Communicated by $\mathrm{K}$. Wysocki

\begin{abstract}
Let $X$ be a compact connected Riemann surface and $\xi$ a square root of the holomorphic cotangent bundle of $X$. Sending any line bundle $L$ over $X$ of order two to the image of $\operatorname{dim} H^{0}(X, \xi \otimes L)-\operatorname{dim} H^{0}(X, \xi)$ in $\mathbb{Z} / 2 \mathbb{Z}$ defines a quadratic form on the space of all order two line bundles. We give a topological interpretation of this quadratic form in terms of index of vector fields on $X$.
\end{abstract}

2000 Mathematics subject classification: primary 14F10, 57R25, 57R15.

\section{The Arf function for a theta characteristic}

Let $X$ be a compact connected Riemann surface of genus $g$. The holomorphic cotangent bundle of $X$ will be denoted by $K_{X}$. Let $\xi$ be a holomorphic line bundle over $X$ such that $\xi^{\otimes 2}$ is holomorphically isomorphic to $K_{X}$. A line bundle with this property is known as a theta characteristic of $X$. Since the degree of $K_{X}$ is even, $X$ has a theta characteristic.

There are exactly $2^{2 g}$ theta characteristics of $X$, where $g$ is the genus of $X$. Indeed, if $L$ is a holomorphic line bundle over $X$ of order two, that is, $L^{\otimes 2}$ is isomorphic to the trivial line bundle, then $\xi \otimes L$ is also a theta characteristic, provided $\xi$ is one. It is easy to see that this action of the order two line bundles on the theta characteristics is free and transitive. In other words, the collection of all theta characteristics of $X$, which we will denote by $S(X)$, is an affine space for the collection of line bundle of order two. Note that the collection of all line bundle of order two, which we will denote by $J_{2}(X)$, is a vector space over $\mathbb{Z} / 2 \mathbb{Z}$ of dimension $2 g$.

(C) 2004 Australian Mathematical Society 1446-7887/04 $\$$ A2.00+0.00 
On $J_{2}(X)$ there is a bilinear form known as the Weil pairing (see [Mul, page 183]). The Weil pairing

$$
\theta_{2}: J_{2}(X) \otimes J_{2}(X) \rightarrow \mathbb{Z} / 2 \mathbb{Z}
$$

is antisymmetric (hence symmetric as the field is $\mathbb{Z} / 2 \mathbb{Z}$ ). Note that in [Mul], the image of $\theta_{2}$ is identified with \pm 1 by sending 1 and -1 to $0 \in \mathbb{Z} / 2 \mathbb{Z}$ and $1 \in \mathbb{Z} / 2 \mathbb{Z}$ respectively. We recall a topological description of the pairing $\theta_{2}$.

The $\mathbb{Z} / 2 \mathbb{Z}$ vector space $J_{2}(X)$ is identified with $H^{1}(X, \mathbb{Z} / 2 \mathbb{Z})$. With this identification, $\theta_{2}$ is simply the cup product on $H^{1}(X, \mathbb{Z} / 2 \mathbb{Z})$. It is easy to see that $J_{2}(X)$ is identified with $\operatorname{Hom}\left(H_{1}(X, \mathbb{Z}), \pm 1\right)$. Indeed, using the natural projection $\pi_{1}(X) \rightarrow$ $H_{1}(X, \mathbb{Z})$ an element in $\operatorname{Hom}\left(H_{1}(X, \mathbb{Z}), \pm 1\right)$ gives a character of order two of the fundamental group $\pi_{1}(X)$. A character of $\pi_{1}(X)$ gives a flat line bundle. Since the above character is of order two, the holomorphic line bundle defined by the corresponding flat line bundle is also of order two. By the above isomorphism of $J_{2}(X)$ with $\operatorname{Hom}\left(H_{1}(X, \mathbb{Z}), \pm 1\right) \cong H^{1}(X, \mathbb{Z} / 2 \mathbb{Z})$, the cup product

$$
H^{1}(X, \mathbb{Z} / 2 \mathbb{Z}) \otimes H^{1}(X, \mathbb{Z} / 2 \mathbb{Z}) \rightarrow H^{2}(X, \mathbb{Z} / 2 \mathbb{Z})=\mathbb{Z} / 2 \mathbb{Z}
$$

translates to the Weil pairing $\theta_{2}$ defined in (1.1).

Take $\xi \in S(X)$, a theta characteristic. Define

$$
\omega_{\xi}: J_{2}(X) \rightarrow \mathbb{Z} / 2 \mathbb{Z}
$$

by $L \mapsto \operatorname{dim} H^{0}(X, \xi \otimes L)-\operatorname{dim} H^{0}(X, \xi) \in \mathbb{Z} / 2 \mathbb{Z}$. The bilinear form associared with the quadratic form $\omega_{\xi}$ in (1.2) coincides with the Weil pairing in (1.1). In other words, for any pair $L_{1}, L_{2} \in J_{2}(X)$ the identity

$$
\omega_{\xi}\left(L_{1} \otimes L_{2}\right)-\omega_{\xi}\left(L_{1}\right)-\omega_{\xi}\left(L_{2}\right)=\theta_{2}\left(L_{1}, L_{2}\right)
$$

is valid [Mu2, page $182,(\star)]$. A function on $J_{2}(X)$ satisfying the identity (1.3) is known as an Arf function [Na, page 93]. In particular, $\omega_{\xi}$ is an Arf function. Any Arf function is of the form $\omega_{\xi}$ for some $\xi \in S(X)$ [Na, page 100, Theorem 10.1].

We will give an alternative description of $\omega_{\xi}$ using the notion of index of a vector field on $X$.

\section{Vector fields and Arf function}

We continue with the notation of the preceding section. Take $\xi \in S(X)$. Take a meromorphic section $s$ of the line bundle $\xi$ which is not identically zero. Therefore, $s \otimes s$ is a meromorphic section of $K_{X}$. In other words, $\hat{s}:=s \otimes s$ is a meromorphic one form of $X$ which is not identically zero. 
Now, since $\hat{s}$ is not identically zero, it defines a meromorphic section of the holomorphic tangent bundle $T X$. Indeed, since $T X \cong K_{X}^{*}$, we have a (unique) meromorphic section $\tau$ of $T X$ defined by the condition that the evaluation $\tau(\hat{s})$ is the constant function 1 on $X$. In particular, the zeros (respectively, poles) of $\hat{s}$ becomes poles (respectively, zeros) of $\tau$. Let $C \subset X$ be the union of all the zeros and poles of $\tau$.

Since $X$ is a compact connected oriented smooth manifold of (real) dimension two, using Poincaré duality we have $H^{1}(X, \mathbb{Z} / 2 \mathbb{Z}) \cong H_{1}(X, \mathbb{Z} / 2 \mathbb{Z})$. Using this isomorphism, the quadratic form $\omega_{\xi}$ on $J_{2}(X)=H^{1}(X, \mathbb{Z} / 2 \mathbb{Z})$ defined in (1.2) would be considered as a quadratic form on $H_{1}(X, \mathbb{Z} / 2 \mathbb{Z})$.

Take any homology class $c \in H_{1}(X, \mathbb{Z} / 2 \mathbb{Z})$. Let $\gamma$ be a smooth oriented loop (that is, a $C^{\infty}$ immersion of the circle $S^{1}=\left\{(x, y) \in \mathbb{R}^{2} \mid x^{2}+y^{2}=1\right\}$ ) in $X \backslash C$ representing the homology class $c$. Since the set $C$ of poles and zeros of the section $\tau$ is finite, such a loop exists. Since $c$ is a homology class with coefficients in $\mathbb{Z} / 2 \mathbb{Z}$, if we reverse the orientation of $\gamma$ then also it represents $c$.

Let index $(\tau, \gamma) \in \mathbb{Z}$ be the index of the vector field $\tau$ on $X \backslash C$ for the oriented loop $\gamma$. We recall the definition of index. If the vector field $\tau$ rotates $n$ times clockwise with respect to the tangent vectors of the curve $\gamma$ (recall that $\gamma$ is an immersion), then index $(\tau, \gamma)=n+1$. For any $p \in S^{1}$, the quotient of the (real) nonzero tangent space $T_{\gamma(p)} X-\{0\}$ by the multiplication action of $\mathbb{R}^{+}$can be identified with $S^{1}=\left\{(x, y) \in \mathbb{R}^{2} \mid x^{2}+y^{2}=1\right\}$ by sending $(1,0) \in S^{1}$ to the tangent vector $\gamma^{\prime}(p)$ along the loop $\gamma$. Using this identification, $\tau$ defines a map from $S^{1}$ to $S^{1}$. The above integer $n$ is the degree of this map.

Henceforth, by index $(\tau, \gamma)$ we will always mean the image in $\mathbb{Z} / 2 \mathbb{Z}$ of the above constructed number.

Note that each pole or zero of $\tau$ is of even order. Therefore, although $\gamma$ is a loop in $X \backslash C$, the dependence of index $(\tau, \gamma)$ on $\gamma$ factors through the image of $\gamma$ in $H_{1}(X, \mathbb{Z} / 2 \mathbb{Z})$. In other words, if a loop $\gamma^{\prime}$ represents a homology class in the kernel of the natural homomorphism $H_{1}(X \backslash C, \mathbb{Z} / 2 \mathbb{Z}) \rightarrow H_{1}(X, \mathbb{Z} / 2 \mathbb{Z})$ (induced by the inclusion map of $X \backslash C$ in $X$ ), then index $\left(\tau, \gamma^{\prime}\right)=0 \in \mathbb{Z} / 2 \mathbb{Z}$.

If $s$ and $s^{\prime}$ are two meromorphic sections of $\xi$, then we have a one-parameter family of meromorphic sections of $\xi$ defined by $\lambda \mapsto s_{\lambda}:=\lambda s+(1-\lambda) s^{\prime}$, where $\lambda \in \mathbb{C}$. So, we have $s_{1}=s$ and $s_{0}=s^{\prime}$. Note that index of a vector field along a loop is a topological invariant. In particular, it does not change under continuous deformations of the vector field. Let $\tau^{\prime}$ be the meromorphic vector field constructed using $s^{\prime}$. Now in view of the above remark that the dependence of index $(\tau, \gamma)$ on $\gamma$ factors through the image of $\gamma$ in $H_{1}(X, \mathbb{Z} / 2 \mathbb{Z})$ it follows immediately that index $(\tau, \gamma)=\operatorname{index}\left(\tau^{\prime}, \gamma\right)$.

Consequently, index $(\tau, \gamma) \in \mathbb{Z} / 2 \mathbb{Z}$ depends only on $\xi$ and $c$. In other words, we are justified in using the notation index $(\xi, c)$ in place of index $(\tau, \gamma)$.

Our aim here is to prove the following theorem. 
THEOREM 2.1. The two elements in $\mathbb{Z} / 2 \mathbb{Z}$, namely $\omega_{\xi}(c)$ and index $(\xi, c)$, coincide.

ProOF. Let $X_{T}$ be a holomorphic family of compact connected Riemann surfaces with a theta characteristic parameterized by a complex manifold $T$. In other words, $X_{T}$ is a complex manifold equipped with a holomorphic submersion $\pi: X_{T} \rightarrow T$ and a holomorphic line bundle $\xi_{T}$ over $X_{T}$ such that for any point $t \in T$, the fiber $X_{t}:=\pi^{-1}(t)$ is a compact connected Riemann surface and the restriction of the line bundle $\xi_{T}$ to $X_{t}$ is a theta characteristic of $X_{t}$. The restriction of $\xi_{T}$ to $X_{t}$ will be denoted by $\xi_{t}$.

A basic theorem due to Atiyah and Mumford says that if the parameter space $T$ is connected then the image of $\operatorname{dim} H^{0}\left(X_{t}, \xi_{t}\right)$ in $\mathbb{Z} / 2 \mathbb{Z}$ is independent of $t$ (see [At, page 28, Theorem 1], [Mu2, page 184, Theorem]). In other words, the parity of $\operatorname{dim} H^{0}\left(X_{t}, \xi_{t}\right)$ remains constant over $T$, provided $T$ is connected.

Now suppose that family $X_{T}$ of Riemann surfaces are equipped with a choice of a first homology class with coefficients in $\mathbb{Z} / 2 \mathbb{Z}$. In other words, for each $t \in T$ we have $\gamma_{t} \in H_{1}\left(X_{t}, \mathbb{Z} / 2 \mathbb{Z}\right)$ with the property that for every contractible open subset $U$ of $T$, the homology class

$$
\gamma_{t^{\prime}} \in H_{1}\left(X_{t^{\prime}}, \mathbb{Z} / 2 \mathbb{Z}\right) \cong H_{1}\left(\pi^{-1}(U), \mathbb{Z} / 2 \mathbb{Z}\right),
$$

where $t^{\prime} \in U$, is independent of $t^{\prime}$. Note that the condition that $U$ is contractible ensures that the homomorphism $H_{1}\left(X_{t^{\prime}}, \mathbb{Z} / 2 \mathbb{Z}\right) \rightarrow H_{1}\left(\pi^{-1}(U), \mathbb{Z} / 2 \mathbb{Z}\right)$ induced by the inclusion map of $X_{t^{\prime}}$ in $\pi^{-1}(U)$ is an isomorphism. Since the index of a vector fields is a topological invariant, in a continuous family of smooth vector fields and loops the index remains unchanged. Therefore, if the parameter space $T$ is connected, the index index $\left(\xi_{t}, \gamma_{t}\right) \in \mathbb{Z} / 2 \mathbb{Z}$ is independent of $t \in T$.

Consequently, both $\omega_{\xi}(c)$ and index $(\xi, c)$ are invariant under deformations of the Riemann surface equipped with choices of a theta characteristic and a homology class.

Now, the moduli space of compact Riemann surfaces of genus $g$ is connected. Therefore, in order to show the equality $\omega_{\xi}(c)=$ index $(\xi, c) \in \mathbb{Z} / 2 \mathbb{Z}$, it suffices to show that the equality holds for just one particular Riemann surface.

We will show that the equality $\omega_{\xi}(c)=\operatorname{index}(\xi, c)$ is valid for a hyperelliptic Riemann surface. This, in view of the above observation, would complete the proof of the theorem.

A hyperelliptic Riemann surface is a double cover of the complex projective line $\mathbb{C P}^{1} \cong \mathbb{C} \cup\{\infty\}$. Fix $2 g+2$ distinct points

$$
\left\{z_{1}, z_{2}, \ldots, z_{2 g+1}, z_{2 g+2}\right\} \subset \mathbb{C} .
$$

This gives a hyperelliptic Riemann surface $X$ defined by the polynomial equation

$$
P(x, y):=y^{2}-\prod_{i=1}^{2 g+2}\left(x-z_{i}\right)=0
$$


where $z_{i}$ are as above. Therefore, we have a degree two map

$$
f: X \rightarrow \mathbb{C P}^{1}
$$

which is ramified exactly over the points $\left\{z_{1}, z_{2}, \ldots, z_{2 g+2}\right\}$. The map $f$ sends a point $(x, y)$ to $x$.

We will now recall an explicit description of all the theta characteristics of this Riemann surface $X$. The details can be found in [Mu2, page 190-191].

Let $\mathscr{O}_{\mathrm{CP}}(1)$ be the (unique) line bundle over $\mathbb{C P}^{1}$ of degree one. Set

$$
\eta:=f^{*} \mathscr{O}_{\mathrm{CPI}}(1)
$$

where $f$ is as in (2.2). So $\eta$ is a holomorphic line bundle over $X$ of degree two. Note that $\eta \cong \mathscr{O}_{X}\left(2 f^{-1}\left(z_{i}\right)\right)$ for each $i \in[1,2 g+2]$ and for each $z \in \mathbb{C} \mathbb{P}^{1} \backslash\left\{z_{1}, z_{2}, \ldots, z_{2 g+2}\right\}$ we have $\eta \cong \mathscr{O}_{X}\left(f^{-1}(z)\right.$ ). (Here $f^{-1}$ denotes the set theoretic inverse (as opposed to the scheme theoretic inverse).)

Take an integer $l \in\{0,1, \ldots,[(g-1) / 2]-1,[(g-1) / 2]\}$ and a subset $S \subset$ $\left\{z_{1}, z_{2}, \ldots, z_{2 g+2}\right\}$ of cardinality $g-1-2 l$. For such a pair $\{l, S\}$ let

$$
\xi(l, S):=\mathscr{O}_{X}\left(f^{-1}(S)\right) \otimes \eta^{\otimes l}
$$

be the holomorphic line bundle over $X$ of degree $g-1$.

For any subset $S \subset\left\{z_{1}, z_{2}, \ldots, z_{2 g+2}\right\}$ of cardinality $g+1$ and $l=-1$, let

$$
\xi(l, S)=\xi(-1, S):=\mathscr{O}_{X}\left(f^{-1}(S)\right) \otimes \eta^{*}
$$

be the holomorphic line bundle over $X$ of degree $g-1$.

Each line bundle $\xi(l, S)$, where

$$
l \in\{-1,0,1, \ldots,[(g-1) / 2]\} \text { and } S \subset\left\{z_{1}, z_{2}, \ldots, z_{2 g+2}\right\}
$$

of cardinality $g-1-2 l$, has the property that $\xi(l, S) \otimes \xi(l, S)$ is isomorphic to $K_{X}$. In other words, $\xi(l, S)$ is a theta characteristic. If $\xi \in S(X)$ is a theta characteristic of $X$, then $\xi$ is isomorphic to $\xi(l, S)$ for some pair $\{l, S\}$. Furthermore, if $\xi(l, S) \cong \xi\left(l^{\prime}, S^{\prime}\right)$, where the pairs $\{l, S\}$ and $\left\{l^{\prime}, S^{\prime}\right\}$ satisfy the above numerical conditions, then the following two conditions

(1) $l=-1=l^{\prime}$ and

(2) $S^{\prime}=\left\{z_{1}, z_{2}, \ldots, z_{2 g+2}\right\} \backslash S$,

are valid (see [Mu2, page 191]).

Set $\delta=f^{-1}(\infty) \subset X$. So $\delta$ consists of two distinct points of $X$ (the map $f$ in (2.2) is unramified over the point $\left.\infty \in \mathbb{C P}^{1}\right)$, and the line bundle $\mathscr{O}_{X}(\delta)$ is isomorphic to $\eta$. 
Given $\{l, S\}$ as above, we will construct a meromorphic section of $K_{X}$ with zeros of order two at each $z \in S$ and a zero (respectively, pole) of order $2 l$ (respectively, 2) for $l$ nonnegative (respectively, for $l=-1$ ) at each point of the set $\delta$.

Recall the polynomial $P(x, y)$ in (2.1) defining $X$. Consider the meromorphic one-form

$$
\omega(\{l, S\}):=\frac{d x}{y} \prod_{z \in S}(x-z)
$$

on $X$. Note that if $z \in S$, then $d x$ has zero of order one at the point $f^{-1}(z)$ (since $f$ in (2.2) is ramified over $z$ ), the function $(x-z)$ has a zero of order two at the point $f^{-1}(z)$ (since $f$ is ramified over $z$ ), and $y$ has pole of order one at $f^{-1}(z)$. Therefore, $\omega(\{l, S\})$ has a zero of order two at $f^{-1}(z)$ if $z \in S$. If $z \in\left\{z_{1}, z_{2}, \ldots, z_{2 g+2}\right\} \backslash S$, then for the same reasons $\omega(\{l, S\})$ is regular (it has neither zero nor pole) at $f^{-1}(z)$.

It is also clear that if $l \geq 0$, then $\omega(\{l, S\})$ has a zero of order $2 l$ at both the points of the subset $\delta$ of $X$. Indeed, for each $z \in S$, the function $x-z$ has a pole of order one at each point of $\delta$. From (2.1) it follows that function $y$ has a pole of order $g+1$ at each point of $\delta$. Finally, since $d x$ has a pole of order two at each point of $\delta$, it follows that $\omega(\{l, S\})$ has a zero of order $-(g-1-2 l)+(g+1)-2=2 l$ at each point of $\delta$.

If $l=-1$, then $\omega(\{l, S\})$ has a pole of order 2 at each of the points of $\delta$. The form $\omega(\{l, S\})$ does not have any other pole or zero.

Therefore, if $D$ is the divisor on $X$ defined by the meromorphic section $\omega(\{l, S\})$, then $D$ is of the form $2 D^{\prime}$ with $\mathscr{O}_{X}\left(D^{\prime}\right) \cong \xi(l, S)$. Indeed, since the line bundle $\mathscr{O}_{X}(\delta)$ is isomorphic to $\eta$, it follows immediately from the definition of $\xi(l, S)$ that $\xi(l, S)$ is isomorphic to the line bundle $\mathscr{O}_{X}\left(D^{\prime}\right)$.

Take any $\xi=\xi(l, S) \in S(X)$ and $c \in H_{1}(X, \mathbb{Z} / 2 \mathbb{Z})$. From the above observation that the divisor of $\omega(\{l, S\})$ is twice the divisor of a section of $\xi$ it follows immediately that to compute index $(\xi, c)$ we can use the meromorphic vector field defined by the one-form $\omega(\{l, S\})$. In other words, in our earlier notation, we can take $\tau$ to be the meromorphic vector field defined by $\omega(\{l, S\})$.

The order two lines bundles over $X$, namely $J_{2}(X)$, is generated by line bundles of the form $\mathscr{O}_{X}\left(f^{-1}\left(z_{i}\right)-f^{-1}\left(z_{j}\right)\right)$, where $\left\{z_{i}, z_{j}\right\} \subset\left\{z_{1}, z_{2}, \ldots, z_{2 g+2}\right\}$. Note that

$$
\begin{array}{r}
\mathscr{O}_{X}\left(f^{-1}\left(z_{i}\right)-f^{-1}\left(z_{j}\right)\right) \otimes \mathscr{O}_{X}\left(f^{-1}\left(z_{i}\right)-f^{-1}\left(z_{j}\right)\right) \\
\cong \mathscr{O}_{X}\left(2 f^{-1}\left(z_{i}\right)\right) \otimes \mathscr{O}_{X}\left(2 f^{-1}\left(z_{j}\right)\right)^{*} \cong \eta \otimes \eta^{*} .
\end{array}
$$

In other words, we have $\mathscr{\theta}_{X}\left(f^{-1}\left(z_{i}\right)-f^{-1}\left(z_{j}\right)\right) \in J_{2}(X)$.

Take a pair $\{l, S\}$ as above. Take a point $z \in S$ and $z^{\prime} \in\left\{z_{1}, z_{2}, \ldots, z_{2 g+2}\right\} \backslash S$. Define $S^{\prime}$ to be $S \cup\left\{z^{\prime}\right\} \backslash\left\{z^{\prime}\right\}$. From the definition of $\xi(l, S)$ it follows immediately that

$$
\xi\left(l, S^{\prime}\right) \cong \xi(l, S) \otimes \mathscr{O}_{X}\left(f^{-1}\left(z^{\prime}\right)-f^{-1}(z)\right)
$$


If we set $S_{1}=S \cup\left\{z^{\prime}, z^{\prime \prime}\right\}$, where $\left\{z^{\prime}, z^{\prime \prime}\right\} \subset\left\{z_{1}, z_{2}, \ldots, z_{2 g+2}\right\} \backslash S$ are two distinct points, then

$$
\xi\left(l-1, S_{1}\right) \cong \xi(l, S) \otimes \mathscr{O}_{X}\left(f^{-1}\left(z^{\prime}\right)+f^{-1}\left(z^{\prime \prime}\right)-f^{-1}(\infty)\right) .
$$

Note that $\mathscr{O}_{X}\left(f^{-1}\left(z^{\prime}\right)+f^{-1}\left(z^{\prime \prime}\right)-f^{-1}(\infty)\right)$ is a line bundle of order two. Similarly, if $S_{2}=S \backslash\{z, \tilde{z}\}$, where $\{z, \tilde{z}\} \subset S$ are two distinct points, then

$$
\xi\left(l+1, S_{2}\right) \cong \xi(l, S) \otimes \mathscr{O}_{X}\left(f^{-1}(\infty)-f^{-1}(z)-f^{-1}(\tilde{z})\right) .
$$

Note that the isomorphism in (2.6) follows from (2.5) by interchanging $S$ and $S_{1}$. The isomorphisms in (2.4), (2.5) and (2.6) together describe the action of $J_{2}(X)$ on $S(X)$, the theta characteristics on $X$.

We know that $\operatorname{dim} H^{0}(X, \xi(l, S))=l+1$ for any pair $\{l, S\}$ of the above type [Mu2, page 191]. Since the action of $J_{2}(X)$ on $S(X)$ is described by the isomorphisms in (2.4), (2.5) and (2.6), we therefore have an explicit description of the quadratic form $\omega_{\xi}$ defined in (1.2).

As before, take a point $z \in S$ and $z^{\prime} \in\left\{z_{1}, z_{2}, \ldots, z_{2 g+2}\right\} \backslash S$. Let $\beta$ denote the tautological meromorphic section of $\mathscr{O}_{X}\left(f^{-1}\left(z^{\prime}\right)-f^{-1}(z)\right)$ given by the constant function 1. So $\beta$ has a pole of order one at $f^{-1}(z)$ and a zero of order one at $f^{-1}\left(z^{\prime}\right)$. From the definition of $\omega(\{l, S\})$ in (2.3) it follows immediately that

$$
\omega\left(\left\{l, S^{\prime}\right\}\right)=\omega(\{l, S\}) \otimes \beta \otimes \beta .
$$

The tensor product $\beta \otimes \beta$ which is a meromorphic function on $X$ (since the line bundle $\mathscr{O}_{X}\left(f^{-1}\left(z^{\prime}\right)-f^{-1}(z)\right) \otimes \mathscr{O}_{X}\left(f^{-1}\left(z^{\prime}\right)-f^{-1}(z)\right)$ is trivial) can also be described as follows. Consider the rational function $\psi$ on $\mathbb{C P}^{1}$ defined by $\psi(x)=\left(x-z^{\prime}\right) /(x-z)$. The function $\beta \otimes \beta$ on $X$ coincides with $\psi \circ f$, where $f$ is defined in (2.2).

Now, as before, take a pair of distinct points $\left\{z^{\prime}, z^{\prime \prime}\right\} \subset\left\{z_{1}, z_{2}, \ldots, z_{2 g+2}\right\} \backslash S$. Consider the rational function $\phi$ on $\mathbb{C P}^{1}$ defined by $\phi(x)=\left(x-z^{\prime}\right)\left(x-z^{\prime \prime}\right) / x^{2}$. Set $S_{1}=S \cup\left\{z^{\prime}, z^{\prime \prime}\right\}$. It is easy to see that the identity

$$
\omega\left(\left\{l-1, S_{1}\right\}\right)=(\phi \circ f) \cdot \omega(\{l, S\})
$$

is valid, where $f$ is defined in (2.2).

In the previous section we saw that $J_{2}(X)$ is naturally identified with $H_{1}(X, \mathbb{Z} / 2 \mathbb{Z})$. We will now describe this identification explicitly for hyperelliptic Riemann surfaces. By $X$ we will denote the hyperelliptic Riemann surface in (2.2).

Let $D^{2}:=\left\{(x, y) \mid x^{2}+y^{2} \leq 1\right\}$ be the closed unit disk and $\stackrel{\circ}{D}^{2}:=\{(x, y) \mid$ $\left.x^{2}+y^{2}<1\right\}$ the open disk, which is the interior of $D^{2}$. The boundary $D^{2} \backslash \dot{D}^{2}$ will be denoted by $S^{1}$. 
Take a pair of distinct points $\left\{z, z^{\prime}\right\} \subset\left\{z_{1}, z_{2}, \ldots, z_{2 g+2}\right\}$. Let $\psi_{0}: D^{2} \rightarrow \mathbb{C}$ be an $C^{\infty}$ embedding of the disk such that

$$
\psi_{0}\left(D^{2}\right) \cap\left\{z_{1}, z_{2}, \ldots, z_{2 g+2}\right\}=\left\{z, z^{\prime}\right\}
$$

and $\left\{z, z^{\prime}\right\} \subset \psi_{0}\left(D^{2}\right)$.

The inverse image $f^{-1}\left(\psi_{0}\left(D^{2}\right)\right)$ is an embedded cylinder in $X$. To prove this first note that the boundary $\psi_{0}\left(S^{1}\right)$ of the image $\psi_{0}\left(D^{2}\right)$ lifts to a loop in $X$. This lifting property of $\psi_{0}\left(S^{1}\right)$ follows from the observation that an embedding

$$
\gamma: S^{1} \rightarrow \mathbb{C P}^{1} \backslash\left\{z_{1}, z_{2}, \ldots, z_{2 g+2}\right\}
$$

lifts to $X$ as a map from $S^{1}$ if and only if the number of point $\left\{z_{i}\right\}$ in some (hence each) component of $\mathbb{C} \mathbb{P}^{1} \backslash \gamma\left(S^{1}\right)$ is even. (Any embedded loop in $\mathbb{C} \mathbb{P}^{1}$ breaks the complement into two connected components; if one component has $j$ points from the set $\left\{z_{i}\right\}$, then the other component has $2 g+2-j$ points of $\left\{z_{i}\right\}$.) Consequently, the inverse image $f^{-1}\left(\psi_{0}\left(D^{2}\right)\right)$ is an embedded cylinder.

Let $\mathrm{I}:=[0,1]$ be the closed interval, $\mathrm{I}:=(0,1)$ the open interval, and $C_{2}:=S^{1} \times \mathrm{I}$ the two dimensional cylinder with boundary. Let $\psi: C_{2} \rightarrow X$ be a $C^{\infty}$ embedding of the cylinder $C_{2}$ into $X$ such that $\psi\left(C_{2}\right)=f^{-1}\left(\psi_{0}\left(D^{2}\right)\right)$. Therefore,

$$
\psi\left(C_{2}\right) \cap\left\{f^{-1}\left(z_{1}\right), f^{-1}\left(z_{2}\right), \ldots, f^{-1}\left(z_{2 g+2}\right)\right\}=\left\{f^{-1}(z), f^{-1}\left(z^{\prime}\right)\right\}
$$

and $\left\{f^{-1}(z), f^{-1}\left(z^{\prime}\right)\right\} \subset \psi\left(S^{1} \times \stackrel{\circ}{\mathrm{I}}\right)$. We saw that $\mathscr{O}_{X}\left(f^{-1}(z)-f^{-1}\left(z^{\prime}\right)\right) \in J_{2}(X)$. The corresponding element in $H_{1}(X, \mathbb{Z} / 2 \mathbb{Z})$ is represented by the image, under the map $\psi$, of $S^{1} \times\{0\}$. Note that $\psi\left(S^{1} \times\{0\}\right)$ and $\psi\left(S^{1} \times\{1\}\right)$ represent the same element in $H_{1}(X, \mathbb{Z} / 2 \mathbb{Z})$.

The element in $H_{1}(X, \mathbb{Z} / 2 \mathbb{Z})$ represented by $\psi\left(S^{1} \times\{0\}\right)$ clearly coincides with the one represented by the lift to $X$ of the loop $\psi_{0}\left(S^{1}\right)$ in $\mathbb{C}$. So both these loops in $X$ represent $\mathscr{O}_{X}\left(f^{-1}(z)-f^{-1}\left(z^{\prime}\right)\right) \in J_{2}(X) \cong H_{1}(X, \mathbb{Z} / 2 \mathbb{Z})$.

Using this observation and the meromorphic forms $\omega(\{l, S\})$ we immediately obtain an explicit description of index $(\xi, c)$. Comparing this with the earlier obtained description of the quadratic form $\omega_{\xi}$ we conclude that $\omega_{\xi}(c)=\operatorname{index}(\xi, c)$.

Therefore, the equality $\omega_{\xi}(c)=\operatorname{index}(\xi, c)$ is valid, provided $X$ is a hyperelliptic Riemann surface. But we already noted that it is enough to prove the equality for just one Riemann surface. Therefore, the proof of the theorem is complete.

\section{References}

[At] M. F. Atiyah, 'Riemann surfaces and spin structures', Ann. Sci. École Nor. Sup. 4 (1971), 47-62. 
[Mul] D. Mumford, Abelian varieties, Tata Institute of Fundamental Research Studies in Mathematics 5 (Oxford University Press, London, 1970).

[Mu2] D. Mumford, 'Theta characteristics of an algebraic curve', Ann. Sci. École Nor. Sup. 4 (1971), 181-192.

[Na] S. M. Natanzon, 'Moduli of Riemann surfaces, Hurwitz-type spaces, and their superanalogs', Russ. Mat. Surveys 54 (1999), 61-117.

School of Mathematics

Tata Institute of Fundamental Research

Homi Bhabha Road

Bombay 400005

India

e-mail: indranil@math.tifr.res.in 
J. Aust. Math. Soc. 76 (2004) 